\title{
PKC- $\delta$ deficiency in B cells displays osteopenia accompanied with upregulation of RANKL expression and osteoclast-osteoblast uncoupling
}

Shangfu Li ${ }^{1,2,3}$, Qiuli Liư ${ }^{4}$, Depeng Wu $u^{1,2,3}$, Tianwei He ${ }^{1,2,3}$, Jinbo Yuan ${ }^{5}$, Heng Qiu ${ }^{5}$, Jennifer Tickner ${ }^{5}$, Song Guo Zheng ${ }^{6}$, Xiaojuan $\mathrm{Li}^{7}$, Jiake $\mathrm{Xu}^{5}$ and Limin Rong ${ }^{1,2,3}$

\begin{abstract}
PKC- $\delta$ is an important molecule for B-cell proliferation and tolerance. B cells have long been recognized to play a part in osteoimmunology and pathological bone loss. However, the role of B cells with PKC- $\delta$ deficiency in bone homeostasis and the underlying mechanisms are unknown. We generated mice with PKC- $\delta$ deletion selectively in $\mathrm{B}$ cells by crossing PKC- $\delta$-loxP mice with CD19-Cre mice. We studied their bone phenotype using micro-CT and histology. Next, immune organs were obtained and analyzed. Western blotting was used to determine the RANKL/ OPG ratio in vitro in B-cell cultures, ELISA assay and immunohistochemistry were used to analyze in vivo RANKL/OPG balance in serum and bone sections respectively. Finally, we utilized osteoclastogenesis to study osteoclast function via hydroxyapatite resorption assay, and isolated primary calvaria osteoblasts to investigate osteoblast proliferation and differentiation. We also investigated osteoclast and osteoblast biology in co-culture with B-cell supernatants. We found that mice with PKC- $\delta$ deficiency in B cells displayed an osteopenia phenotype in the trabecular and cortical compartment of long bones. In addition, PKC- $\delta$ deletion resulted in changes of trabecular bone structure in association with activation of osteoclast bone resorption and decrease in osteoblast parameters. As expected, inactivation of PKC- $\delta$ in B cells resulted in changes in spleen B-cell number, function, and distribution. Consistently, the RANKL/OPG ratio was elevated remarkably in B-cell culture, in the serum and in bone specimens after loss of PKC- $\delta$ in $B$ cells. Finally, in vitro analysis revealed that PKC- $\delta$ ablation suppressed osteoclast differentiation and function but coculture with B-cell supernatant reversed the suppression effect, as well as impaired osteoblast proliferation and function, indicative of osteoclast-osteoblast uncoupling. In conclusion, PKC- $\delta$ plays an important role in the interplay between B cells in the immune system and bone cells in the pathogenesis of bone lytic diseases.
\end{abstract}

\section{Introduction}

Osteoimmunology was an interdisciplinary subject coined by Arron in 2000 to highlight the reciprocal interactions between the skeletal and immune systems ${ }^{1}$.

\footnotetext{
Correspondence: Shangfu Li (lishangfu@mail.sysu.edu.cn) or Jiake Xu (jiake. xu@uwa.edu.au) or Limin Rong (ronglm@mail.sysu.edu.cn)

'Department of Spine Surgery, The Third Affiliated Hospital of Sun Yat-sen University, Guangzhou Guangdong, China

${ }^{2}$ Guangdong Provincial Center for Quality Control of Minimally Invasive Spine Surgery, Guangzhou, China

Full list of author information is available at the end of the article These authors contributed equally: Shangfu Li, Qiuli Liu, Depeng Wu Edited by Y. Shi
}

Given the tight anatomical and physiological coexistence of $\mathrm{B}$ cells and the bone-forming units in the bone marrow, accumulating evidence indicated an important role for B cells in osteoimmunological regulation ${ }^{2}$. Recent findings indicating that $\mathrm{B}$ cells are active regulators of the receptor activator of NF-kB (RANK)/receptor activator of NF- $k B$ ligand (RANKL)/osteoprotegerin (OPG) $\mathrm{axis}^{3-5}$, and that RANKL expression by B cells contributed to ovariectomyinduced bone loss further support this assumption ${ }^{6}$.

Protein kinase $C \delta$ (PKC- $\delta$ ) was first cloned over 30 years ago $^{7}$ and it is notably identified as an essential regulator of peripheral B-cell development and a critical

\section{(-) The Author(s) 2020}

(c) (i) Open Access This article is licensed under a Creative Commons Attribution 4.0 International License, which permits use, sharing, adaptation, distribution and reproduction cc) in any medium or format, as long as you give appropriate credit to the original author(s) and the source, provide a link to the Creative Commons license, and indicate if changes were made. The images or other third party material in this article are included in the article's Creative Commons license, unless indicated otherwise in a credit line to the material. If material is not included in the article's Creative Commons license and your intended use is not permitted by statutory regulation or exceeds the permitted use, you will need to obtain permission directly from the copyright holder. To view a copy of this license, visit http://creativecommons.org/licenses/by/4.0/. 
regulator of immune homeostasis ${ }^{8}$. In 2002 , PKC- $\delta$ null mice, which develop systemic autoimmunity revealed an essential role for this kinase in B-cell homeostasis and tolerance ${ }^{9,10}$. In humans, B cells are considered central in lupus pathogenesis and PKC- $\delta$ deficiency was identified as the first B-cell-related subset of monogenic lupus. Biallelic mutations in PRKCD (the gene that encodes PKC- $\delta$ ) are associated with lupus and lymphoproliferative diseases because PKC- $\delta$ displays proapoptotic activity and is crucial to eliminate self-reactive transitional $\mathrm{B}$ cells ${ }^{11-14}$. These findings further confirmed PKC- $\delta$ as a critical proapopotic molecule essential in B-cell survival and apoptosis.

Bone cells (such as osteoclasts (OCs), osteoblasts (OBs), and osteocytes) and hematopoietic cells share the same microenvironment in the bone marrow and interact with each other to cooperatively regulate the functional activities of the bone system. PKC- $\delta$ deficiency perturbs bone homeostasis by selective uncoupling of Cathepsin $\mathrm{K}$ (CTSK) secretion and ruffled border formation in $\mathrm{OCs}^{15}$, and loss of PKC- $\delta$ protected against LPS-induced osteolysis owing to an intrinsic defect in osteoclastic bone resorption ${ }^{16}$. In addition, PKC modulated the synthesis of nitric oxide by $\mathrm{OBs}^{17}$ and noncanonical Wnt signaling through G-protein-linked PKC- $\delta$ activation promoted bone formation ${ }^{18}$. Moreover, PKC- $\delta$ played an important role in the osteochondral plasticity of the interface between articular cartilage and the osteochondral junction $^{19}$. These studies revealed that PKC- $\delta$ not only played an essential role in immunity but also in skeletal biology. RANKL interacts with two receptors, one functionally called RANK and the other a decoy named OPG. RANKL is a key OC differentiation factor and was found to play an essential role not only in the development of immune organs and bones, but also in autoimmune diseases affecting bone $\mathrm{e}^{20}$. In addition, B-lymphoid lineage cells are a major source of endogenous RANKL in bone marrow and support OC differentiation in vitro ${ }^{21}$. However, the association between PKC- $\delta$ function and RANKL expression in B cells, and its role in bone homeostasis remain unclear.

Our study aimed to investigate the important role of PKC- $\delta$ in B cells and its subsequent effects on $O C$ and $O B$ biology by using a Cre-loxP-based conditional knockout (cKO) technology to selectively inactivate $\mathrm{PKC}-\delta$ in $\mathrm{B}$ cells, which could help to shed more light on our understanding of osteoimmunology-related disease, such as rheumatoid arthritis and osteoporosis.

\section{Results}

PKC- $\delta$ conditional knockout in B cells results in osteopenia and altered bone microstructure in mice

Firstly, we established and confirmed CD19-driven $\mathrm{PKC}-\delta$ deletion in $\mathrm{B}$ cells in mice. We used the conditional PKC- $\delta$ allele in which exon 7 is flanked by loxP sites. Cre-mediated deletion of exon 7 results in a PKC- $\delta$ null allele in B cells (Supplementary Fig. 1a). Efficiency of Cre-mediated deletion of PKC- $\delta$ exon 7 and consequent loss of PKC- $\delta$ expression in B cells was confirmed by DNA PCR for the deleted and floxed alleles (Supplementary Fig. 1b). Further, significant decrease of PKC- $\delta$ mRNA (Supplementary Fig. 1c) and almost absence of protein expression (Supplementary Fig. 1d) in B cells were verified.

To determine the contribution of PKC- $\delta$ cKO in B cells in skeletal development and bone homeostasis, we firstly analyzed the gross appearance of 3-month-old PKC- $\delta$ cKO mice. Interestingly, there were no significant changes in both male and female mice regarding their body weight (Fig. 1a), suggesting CKO mice had a similar body structure to that of WT littermates. We further examined the bone microstructure using microcomputed tomography (micro-CT). Interestingly, micro-CT analysis revealed that $\mathrm{PKC}-\delta$ deficiency changed bone volume and microstructure in both femur (Fig. 1b) and tibia (Fig. 1c) in cKO mice compared to age- and sex-matched WT littermates. In the femur, the percentage of trabecular bone volume versus total volume (BV/TV, Fig. 1bii) and trabecular thickness (Tb.Th, Fig. 1biv) were all significantly reduced in male mice but not in female mice in the trabecular bone. There were a trend of decrease in trabecular number (Tb.N, Fig. 1biii) and a trend of increase in trabecular separation (Tb.Sp, Fig. 1bv); however, no significant statistical differences were found. In the femoral cortical bone, total cortical area (Tt.Ar, Fig. 1bvii) and cortical bone area (Ct.Ar, Fig. 1bviii) were also all significantly reduced in male mice but increased for female mice compared with the WT controls. However, there were no significant changes in cortical area fraction (Ct.Ar/Tt.Ar, Fig. 1bix) and cortical thickness (Ct.Th, Fig. 1bx). In the tibia, for the trabecular bone micro-CT parameters, only BV/TV (Fig. 1cii) was significantly reduced for both male and female mice compared with WT control mice. No significant differences were found in Tb.N (Fig. 1ciii), Tb.Th (Fig. 1civ) and Tb.Sp (Fig. 1cv). For the tibial cortical bone parameters, there were significant decreases in male but not in female mice regarding Tt.Ar (Fig. 1cvii) and Ct.Ar (Fig. 1cviii). However, no significant differences were found in Ct.Ar/ Tt.Ar (Fig. 1cix) and Ct.Th (Fig. 1cx).

Taken together, these findings show there is an osteopenic phenotype in both trabecular bone and cortical bone after PKC- $\delta$ ablation in B cells.

\section{Decreased bone fraction and osteoblasts and increased osteoclasts in trabecular bone of PKC- $\delta$ conditional knockout mice}

To gain further insight into the in vivo osteopenic phenotype of the cKO mice, bone histomorphometry was 


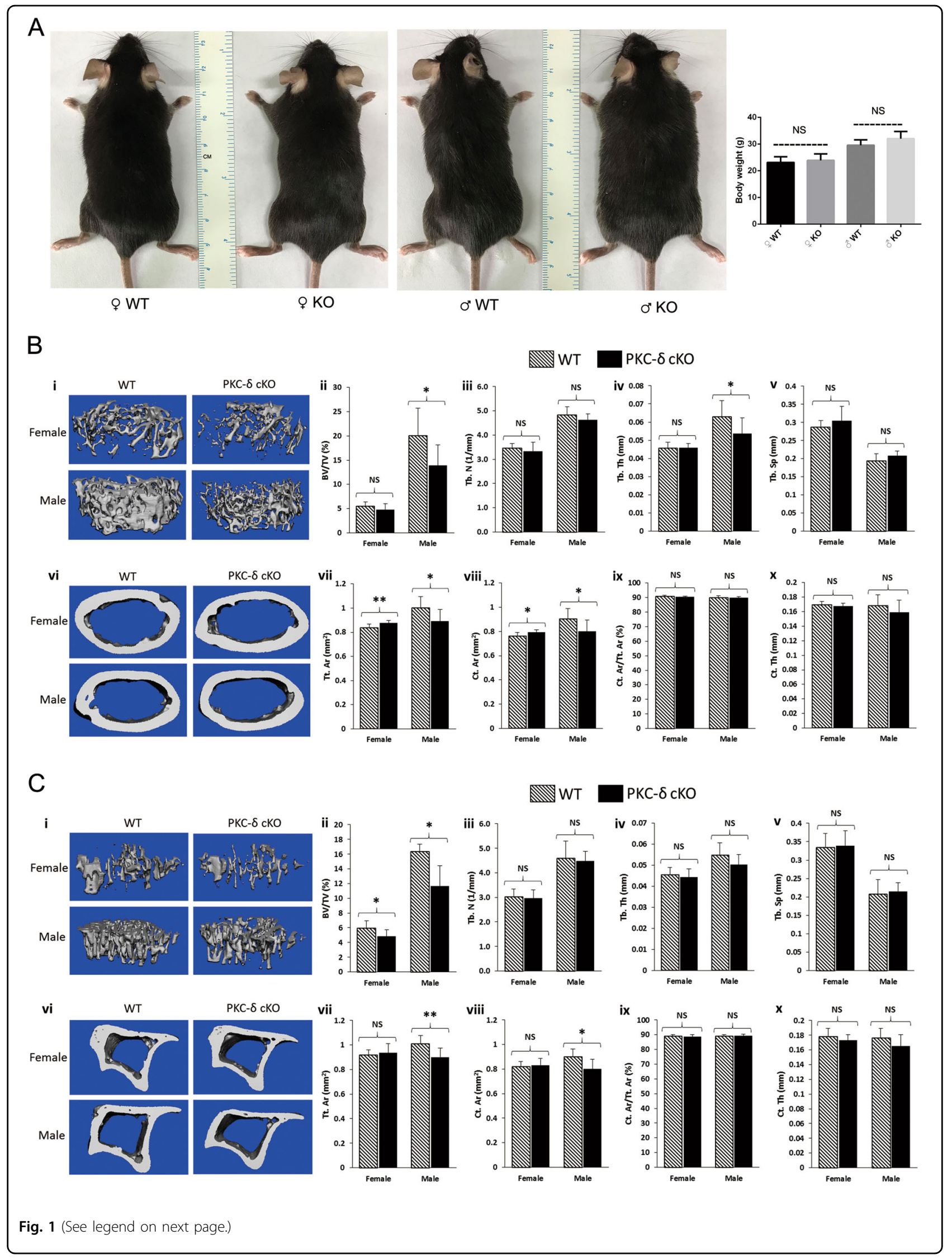


(see figure on previous page)

Fig. 1 Micro-CT analysis of hind limbs revealing an osteoporotic phenotype in 12-week-old mice with PKC- $\delta$ conditional knockout in

B cells. a Representative images and body weight of the mice at 12 weeks of age (WT wild-type, KO knockout). NS = non-significant compared with WT controls; $\mathbf{b}, \mathbf{c}$ Representative 3D reconstructions of trabecular and cortical bone and bone parameters assessed by micro-CT in distal femur ( $\mathbf{b}$ i-x) and proximal tibia (c $\mathrm{i}-\mathrm{x}$ ) in age- and sex-matched WT and PKC- $\delta$ conditional knockout (cKO) mice, respectively ( $\mathrm{male}$ WT $n=6, \mathrm{male} c \mathrm{KO} n=7$, female WT $n=7$, female $c K O n=7$ ). Trabecular bone parameters ( $\mathbf{b}$ ii- $v$ and $\mathbf{c}$ ii-v) are shown as trabecular bone volume fraction (BV/TV, \%; $\mathbf{b}$ ii and c ii), trabecular number (Tb.N, 1/mm; $\mathbf{b}$ iii and $\mathbf{c}$ iii), trabecular thickness (Tb.Th, mm; b iv and $\mathbf{c}$ iv) and trabecular separation (Tb.Sp, mm; $\mathbf{b} \vee$ and $\mathbf{c} v$ ). Micro-CT analysis of cortical bone parameters (b vii-x and $\mathbf{c}$ vii-x) are shown as total cortical area (Tt.Ar, $\mathrm{mm}^{2} ; \mathbf{b}$ vii and $\mathbf{c}$ vii), cortical bone area (Ct.Ar, $\mathrm{mm}^{2} ; \mathbf{b}$ viii and $\mathbf{c}$ viii), cortical area fraction (Ct.Ar $/ \mathrm{Tt} . \mathrm{Ar}, \% ; \mathbf{b}$ ix and $\mathbf{c}$ ix) and cortical thickness (Ct.Th, $\mu \mathrm{m} ; \mathbf{b} \times$ and $\mathbf{c} \times$ ). Data are presented as mean \pm SD. ${ }^{*} p<0.05,{ }^{* *} p<0.01, \mathrm{NS}=$ non-significant compared with WT controls.

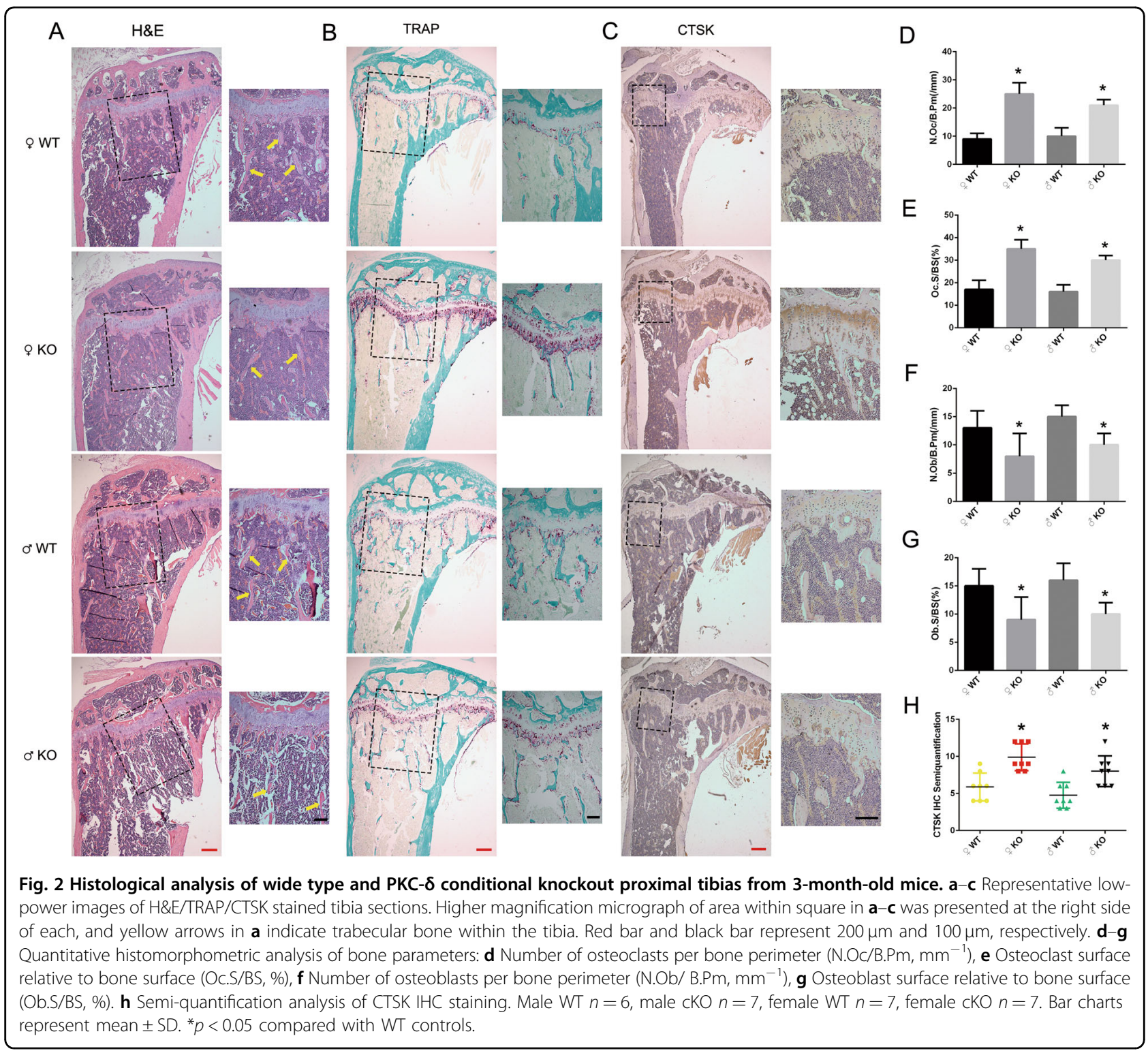

performed on decalcified sections stained with hematoxylin and eosin (H\&E) (Fig. 2a) and for tartrate-resistant acid phosphatase (TRAP) activity (Fig. 2b). Consistent with the bone loss phenotype demonstrated by micro-CT, $\mathrm{H} \& \mathrm{E}$ staining of tibia from 3-month-old cKO mice showed that trabecular bone was greatly reduced when compared to WT mice (Fig. 2a). Bone remodeling is a highly coordinated process requiring bone resorption by $\mathrm{OC}$ and bone formation by $\mathrm{OB}$ and is necessary to maintain mineral homeostasis ${ }^{22}$. We thus further 
investigated $\mathrm{OB}$ and $\mathrm{OC}$ number and distribution in vivo in bone sections to elucidate the osteopenic phenotype. Consistent with the micro-CT findings, analysis of OC parameters using TRAP stained sections (Fig. 2b) revealed that $\mathrm{cKO}$ mice exhibited a significant increase in the number of OCs per bone perimeter (Fig. 2d) and OC surface per bone surface (Fig. 2e). We further verified our finding by examining the expression of CTSK, an important biomarker for OC function, using immunohistochemistry (IHC) staining. As expected, semiquantitative analysis showed increased CTSK activity after inactivation of PKC- $\delta$ in B cells when compared with the WT controls (Fig. 2h). For the in vivo OB parameters, as expected, $H \& E$ staining revealed that $O B$ number per bone perimeter (Fig. 2f) and OB surface per bone surface (Fig. 2g) in the tibia displayed significant decrease when compared with the WT littermates. Osteocalcin IHC staining further verified the reduction of osteoblasts in the trabecular bone in mice with B-cell-specific deletion of PKC- $\delta$ (Supplementary Fig. 2).

Articular cartilage plays a critical role in bone and cartilage homeostasis $^{23}$, previous study showed that PKC$\delta$ is an important regulator of osteochondral plasticity ${ }^{19}$. Next, we examined cartilage changes using Safranin O Fast Green Staining, no significant differences regarding articular cartilage thickness and no osteoarthritis-like features were found as shown for the representative images (Supplementary Fig. 3a). Bone is a dynamic organ composed of organic and inorganic elements ${ }^{24}$. Representative images of von Kossa staining showed decreased inorganic contents in the trabecular bone of PKC- $\delta \mathrm{cKO}$ mice (Supplementary Fig. 3c). No changes to organic components were observed as shown in the representative images of Masson's trichrome staining (Supplementary Fig. 3b).

Collectively, these results suggest that the osteopenic phenotype after cKO of PKC- $\delta$ in B cells was mainly due to $\mathrm{OC}$ and $\mathrm{OB}$ changes.

\section{PKC- $\delta$ deficiency in B cells was accompanied by changes in} $B$-cell number, function, and distribution

PKC- $\delta$ plays an important role in immune organ development and skeletal diseases ${ }^{2,8}$. We further investigated its role in B-cell development and function. It has been reported that PKC- $\delta$ null mice had an increase of B-cell proliferation ${ }^{10}$, B1a cells have been implicated in the development of autoimmune arthritis through RANKL-mediated osteoclastogenesis ${ }^{25}$, but it is not clear whether PKC- $\delta$ deficiency selectively in B cells will affect B1a cells. Hence, we systematically compared the percentage of B1a cells (mainly characterized by expression both of CD19 and CD5) in different immune organs, and investigated changes in the number, distribution, and function of B cells after PKC- $\delta$ depletion. We obtained single-cell suspensions from spleen, lymph nodes, liver, and thymus, then analyzed the percentage of B1a cells by flow cytometry (Fig. 3ai). The step by step gating schedule is shown in Supplementary Fig. 4. Interestingly, we found that the percentage of B1a cells was significantly increased only in spleen after PKC- $\delta$ ablation, especially in male mice (Fig. 3aii). We further evaluated the change of B1a cells function by detecting the important antiinflammatory factor IL-10 (Fig. 3bi), we found that their ability to secrete IL-10 was significantly reduced in B cells with depletion of PKC- $\delta$ (Fig. 3bii). Next, we separately evaluated the total number of spleen cells in PKC- $\delta$ deficient mice, and the number of purified $B$ cells in the spleen. As shown in Fig. 3c, the number of total spleen cells was increased in both female and male mice after PKC- $\delta$ inactivation (Fig. $3 \mathrm{ci}$ ), while the number of purified B cells obtained from the total spleen cells (the absolute value) was significantly increased only in male mice but not in female mice after ablating PKC- $\delta$ (Fig. 3cii). Interestingly, an increased liver weight (Fig. 3ciii) and larger spleen (Fig. 3d) were also observed in male mice but not in female mice after PKC- $\delta$ cKO. Consistently, we also found that the B cells were significantly increased (Fig. 3ei, indicated by red color) after PKC- $\delta$ delection by measuring the fluorescence intensity of B220 (Fig. 3eii), which was expressed by the B-cell lineage from early pro-B cells to mature B cells. Intriguingly, we observed that the B-cell area in the spleen became larger both in male and female mice after PKC- $\delta$ cKO (Fig. 3ei).

Taken together, our data showed that PKC- $\delta$ deficiency in B cells was accompanied by changes in B-cell number, function, and distribution mainly in the spleen.

\section{PKC- $\delta$ deficiency in B cells increased RANKL/OPG ratio}

RANKL/OPG/RANK signaling regulates numerous physiological processes including bone remodeling and lymph node organogenesis ${ }^{20}$. RANKL is best known for its indispensable role in promoting differentiation of mature $\mathrm{OCs}^{26}$. We thus investigated the RANKL/OPG ratio after ablation of PKC- $\delta$ in B cells. Firstly, we assessed the purity of B cells sorted by flow cytometry $(98.7 \%$, Fig. 4a). Next, we performed in vitro spleen-derived B-cell culture. Importantly, we found that the protein expression of RANKL and the ratio of RANKL/OPG were all significantly increased in female and male PKC- $\delta$ cKO mice (Fig. 4b). However, OPG protein expression was almost unchanged after ablation of PKC- $\delta$, indicating that $\mathrm{PKC}-\delta$ deficiency in B cells raised the RANKL/OPG ratio mainly due to the overexpression of RANKL. Furthermore, we collected the serum from 3-month-old mice to determine the concentration of OPG, RANKL and bone turnover markers of $\beta$-CTX and PINP by ELISA. As expected, the level of RANKL in PKC- $\delta$ cKO mice was remarkably increased both for male and female (Fig. 4ci), while there 


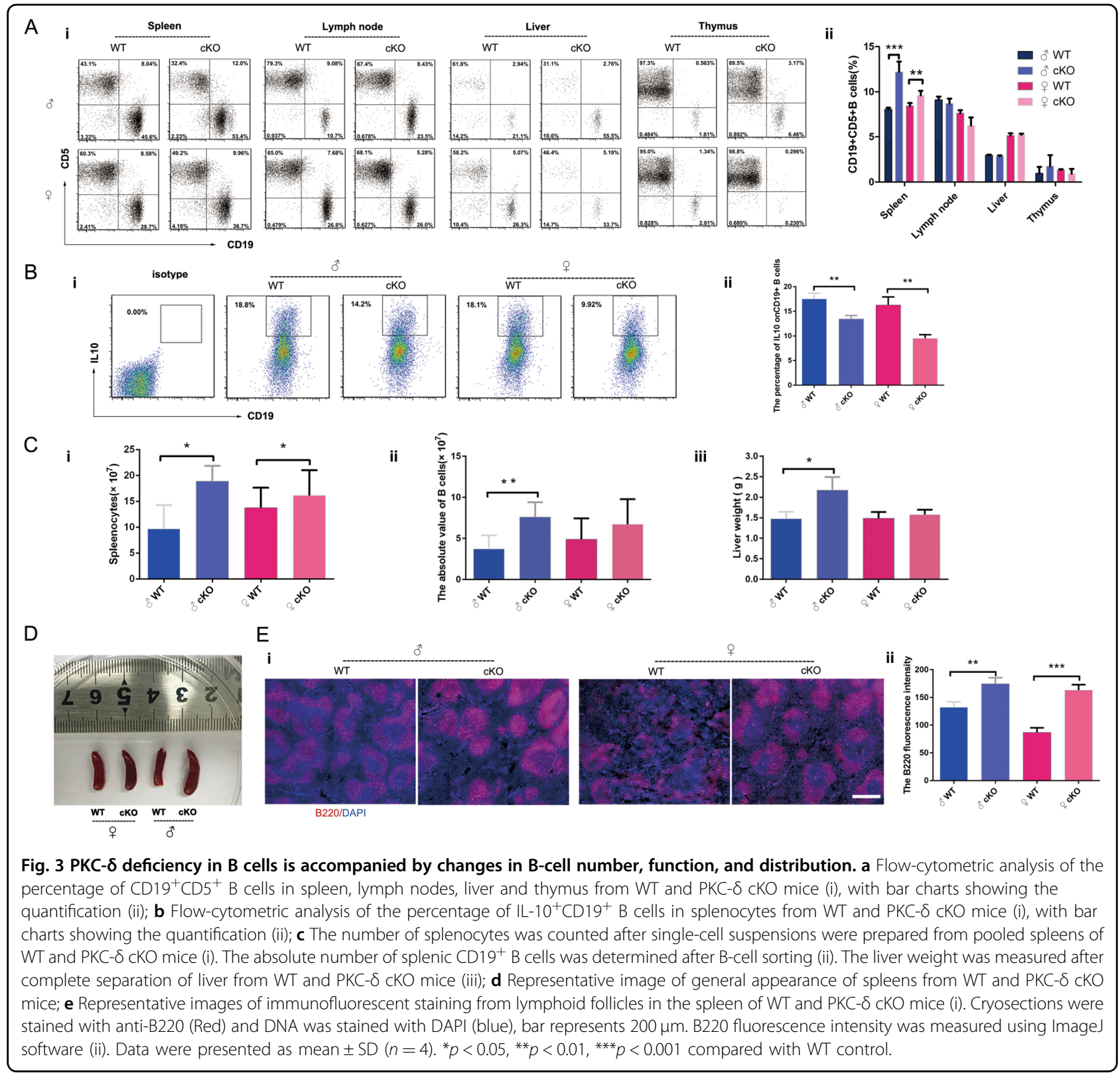

were no significant changes in OPG concentration after deletion of PKC- $\delta$ (Fig. 4cii). Unexpectedly, the bone resorption marker $\beta$-CTX (Fig. 4ciii) and the bone formation marker PINP (Fig. 4civ) were not significantly changed in both female and male mice after PKC- $\delta$ cKO. We next asked whether the elevation of RANKL both in B cells and in the serum would indirectly affect bone tissue, we thus performed RANKL and OPG IHC in tibia sections to verify our hypothesis. As showed in Fig. $4 d-g$, the staining intensity in bone cells, especially OCs, was significantly enhanced for RANKL IHC (Fig. 4d, f) in both female and male mice with ablation of PKC- $\delta$ in B cells. However, OPG staining intensity was unchanged as assessed by semi-quantitative analysis when compared with the WT littermates (Fig. 4e, g).

Accordingly, all these results indicated that $\mathrm{PKC}-\delta$ deficiency in B cells led to increased RANKL/OPG ratio in cell cultures, in the serum and in the trabecular bone, and eventually caused an osteopenic phenotype.

\section{PKC- $\delta$ deletion in B cells resulted in osteoclast-osteoblast uncoupling}

In addition to the increased OCs observed in PKC- $\delta$ cKO mice in vivo, we further examined the effects of PKC- $\delta$ ablation in $\mathrm{B}$ cells on osteoclastogenesis and bone resorption in vitro. Surprisingly, BMMs from PKC- $\delta$ cKO 


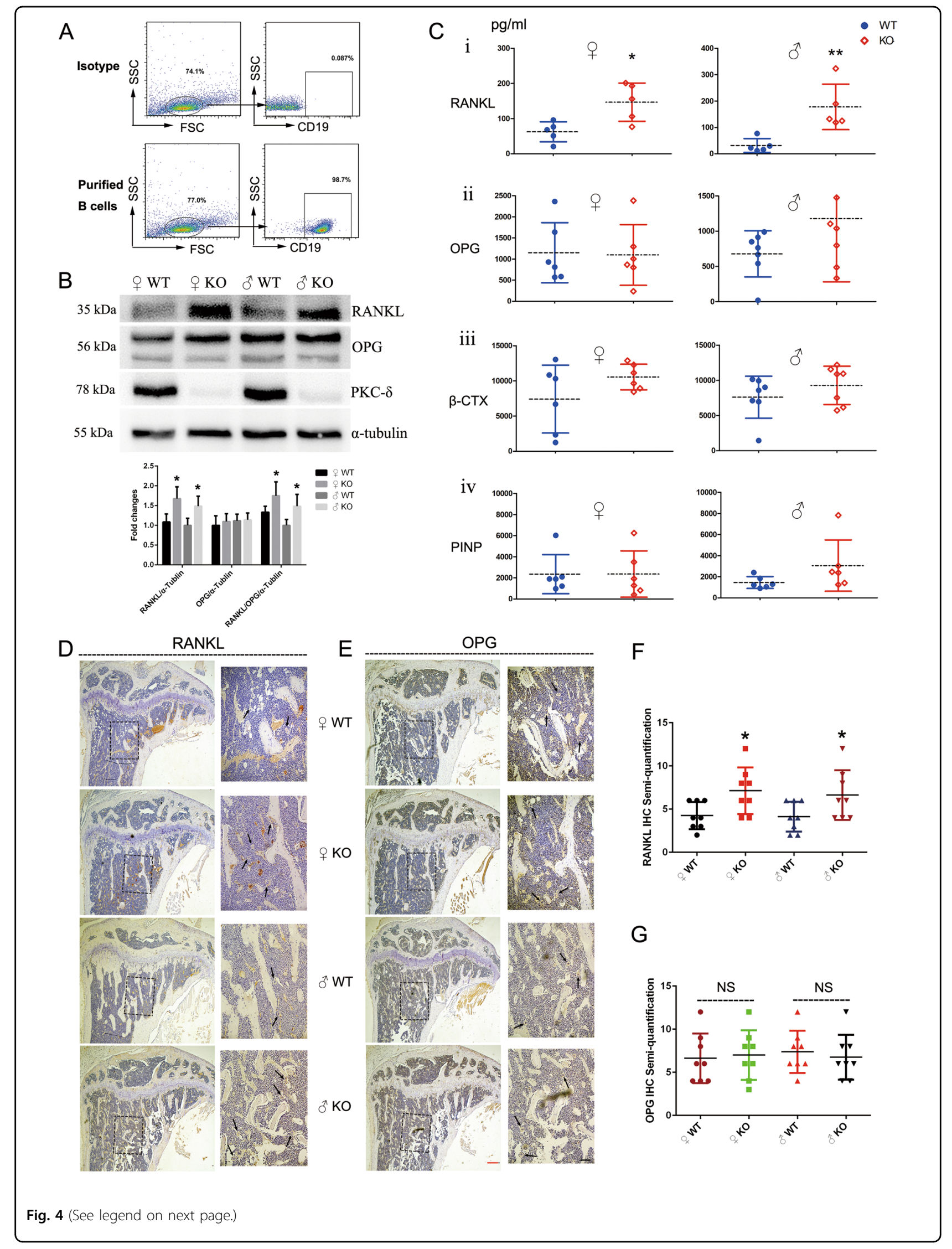


(see figure on previous page)

Fig. 4 PKC- $\delta$ deficiency in B cells elevated RANKL/OPG ratio in both cell culture and serum and increased RANKL expression in the trabecular bone. a Flow-cytometry plots representing the purity of B cells after sorting; $\mathbf{b}$ Expression of PKC- $\delta$, OPG, and RANKL in B cells from WT and PKC- $\delta$ cKO mice was detected by western blotting, with semi-quantitative analysis; $\mathbf{c}$ Blood was obtained from the fundus vein of WT and PKC- $\delta$ CKO mice. The concentrations of RANKL, OPG, $\beta-C T X$, and PINP were determined by ELISA in the serum. Each data point was from an individual mouse and means were indicated by dashed horizontal lines. $\mathbf{d}$, e Representative images of RANKL (d) and OPG (e) immunohistochemistry stained tibia sections of 3-month-old PKC- $\delta$ CKO and age-sex-matched wild-type mice. Higher magnification micrograph of area within square in $\mathbf{d}$, e is presented at the right side of each. Arrows indicate the positive staining of osteoclasts in the trabecular bone within the tibia. Red bar and black bar represent $200 \mu \mathrm{m}$ and $50 \mu \mathrm{m}$, respectively. f, g Semi-quantitative analysis of RANKL (f) and OPG (g) immunohistochemistry staining. Bar charts represent mean $\pm \mathrm{SD} .{ }^{*} p<0.05,{ }^{* *} p<0.01, \mathrm{NS}=$ non-significant compared with WT control group.

produced significantly less OCs than WT controls after induction with $100 \mathrm{ng} / \mathrm{ml}$ RANKL for 7 days (Fig. 5a, b). We further assessed OC function by culturing mature osteoclasts derived from BMMs with $100 \mathrm{ng} / \mathrm{ml}$ RANKL for 7 days on hydroxyapatite-coated plates. Unexpectedly, the area of bone resorbed by PKC- $\delta$ cKO OCs was significantly decreased as compared to WT control (Fig. 5c, d). To confirm these findings, we detected the key genes and their downstream protein expression related to OC differentiation and function. As shown in Fig. 5e, the expression levels of these OC-related genes of nfatcl, ctsk, and calcitonin receptor were all remarkably decreased after stimulating with $100 \mathrm{ng} / \mathrm{ml}$ RANKL for 5 days in mice with deletion of PKC- $\delta$. Moreover, the expression levels of downstream proteins related to $\mathrm{OC}$ bone resorption activity, such as NFATc1, CTSK, and Carbonic Anhydrase II (CAII), were also decreased at 7 days after $100 \mathrm{ng} / \mathrm{ml}$ RANKL stimulation in PKC- $\delta$ cKO mice (Fig. 5f, g). Because PKC- $\delta$ deficiency in B cells led to increased RANKL in both B-cell cultures and in the serum (Fig. $4 \mathrm{a}-\mathrm{C}$ ), we further performed B-cell co-culture experiments with OCs to verify our observations. B cells are prone to die in the culture medium, so we used B-cell supernatant (BS) for the co-culture experiments. As shown in Fig. 5a-d, osteoclastogenesis and bone resorption were all reduced after stimulation with $25 \mathrm{ng} / \mathrm{ml}$ RANKL for 7 days in PKC- $\delta$ cKO mice. However, as expected, BS co-culture significantly enhanced both OC formation and function and, surprisingly, reversed the suppression effect of PKC- $\delta$ deletion in B cells on OC biology.

Because $\mathrm{OC}-\mathrm{OB}$ coupling plays the most important role during bone remodeling ${ }^{22}$, to investigate whether PKC- $\delta$ cKO has an indirect effect on OB biology, we evaluated the regulation of $\mathrm{PKC}-\delta$ in OB proliferation and function. Proliferation rates of OBs derived from cKO mice were significantly reduced at $48 \mathrm{~h}$ (Fig. 6a). Furthermore, OBs derived from calvaria of cKO mice displayed a significant reduction of function in both a reduction in alkaline phosphatase (ALP) staining at days 7 (Fig. 6b, c) and in mineralization activity as evidenced by alizarin red staining at days 21 , respectively. Moreover, the potent promotion effect of bone morphogenetic protein-2 (BMP-2) on osteoblastogenesis was greatly reduced after PKC- $\delta$ ablation as evidenced by alizarin red staining (Fig. 6d, e). We further carried out co-culture experiments with BS, which contained soluble RANKL during osteoblastogenesis. Surprisingly, BS co-culture had no effect on mineralized nodule formation (Fig. 6d, e). Furthermore, we did not observe any stimulation effect of recombinant (soluble) RANKL proteins $(50 \mathrm{ng} / \mathrm{ml}$ and $100 \mathrm{ng} / \mathrm{ml}$ ) on bone formation after treating MC3T3-E1 cells for 7 days (Supplementary Fig. 5). These results revealed that soluble RANKL, unlike membrane-bound RANKL $^{27}$, had no effect on osteoblast formation and function. To further investigate the potential mechanisms of PKC- $\delta$ ablation in suppressing osteoblastogenesis at the molecular level, we firstly detected osteoblast-specific gene transcription and found that the relative mRNA expression of osteoblast transcription factors ocn and run $x 2$ were significantly downregulated by loss of PKC- $\delta$, while colla1 had a trend of reduction without statistically significant difference (Fig. 6f). In addition, the protein expression level of osteoblast-specific RUNX2 was also reduced at day 7 and day 14 during osteoblastogenesis after loss of PKC- $\delta$, moreover, the OB response to BMP-2 stimulation was markedly inhibited by PKC- $\delta$ ablation (Fig. 6g, h). To evaluate whether PKC- $\delta$ cKO suppresses the Wnt signaling pathway, we initially analyzed the expression levels of $\beta$-catenin during osteoblastogenesis and found it decreased at day 7 and day 14 in PKC- $\delta$ cKO mice (Fig. $6 \mathrm{~g}, \mathrm{~h}$ ). Given the fact that GSK-3 $\beta$ induced the phosphorylation and degradation of $\beta$-catenin in the absence of Wnt activation, we therefore investigated whether the impairment of the Wnt/ $\beta$-catenin pathway by PKC- $\delta$ cKO is involved in GSK- $3 \beta$ activation. As shown in Fig. $6 \mathbf{i}-\mathbf{j}$, the phosphorylation of GSK-3 $\beta$ decreased when treated with $\mathrm{LiCl}$ at 0,30 , and $60 \mathrm{~min}$ after PKC- $\delta$ ablation. Moreover, the expression level of TCF, a key transcription factor of the $\mathrm{Wnt} / \beta$-catenin pathway, was also significantly decreased after $\mathrm{LiCl}$ treatment. Collectively, PKC- $\delta$ ablation in B cells suppresses Wnt $/ \beta$-catenin pathways accompanied by phosphorylation of GSK-3 $\beta$.

In summary, $\mathrm{PKC}-\delta$ ablation in $\mathrm{B}$ cells disturbs the OC-OB balance. 


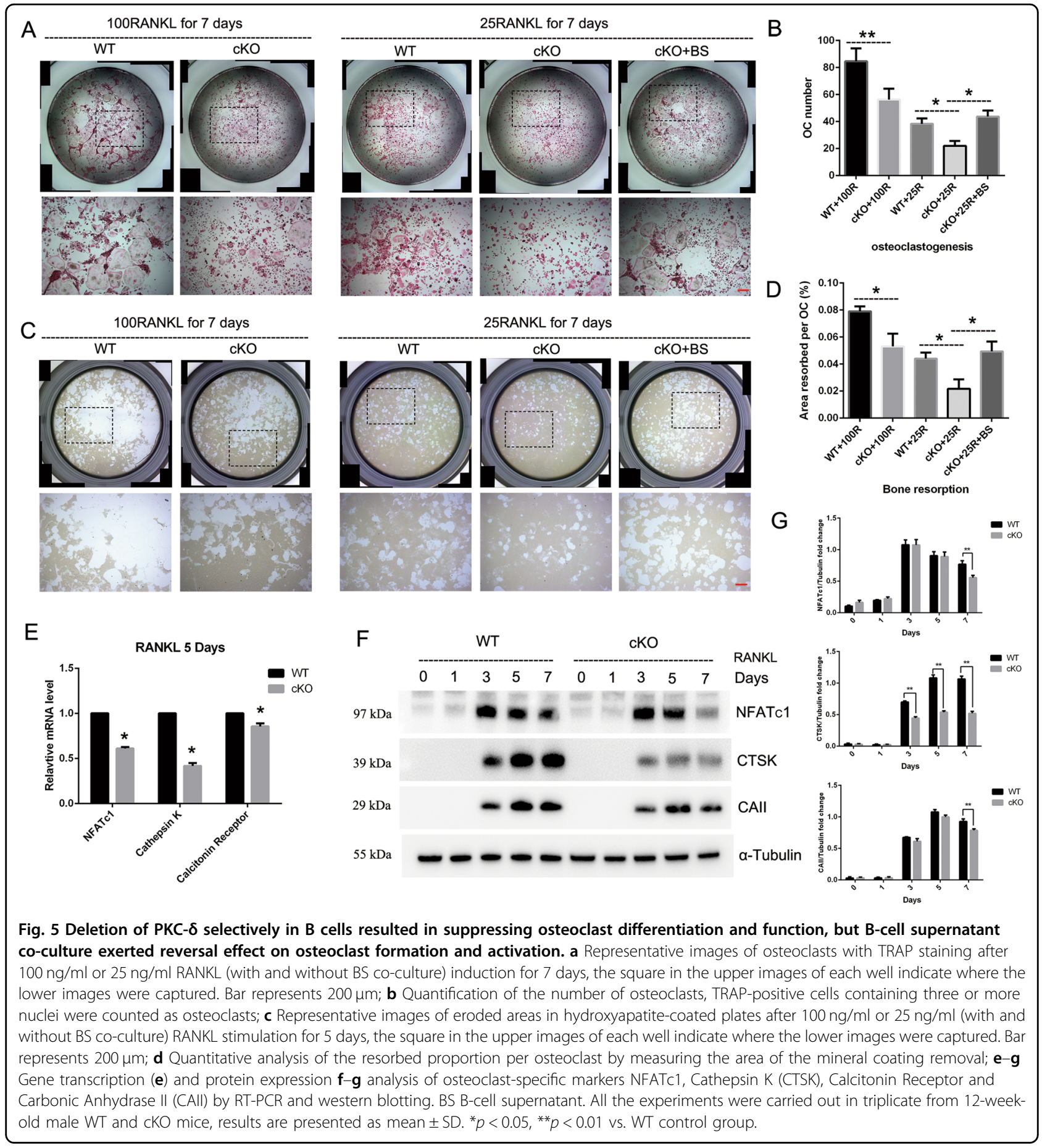

\section{Discussion}

In this study, we found that mice with PKC- $\delta$ deficiency selectively in B cells displayed osteopenia and hyperproliferation of $\mathrm{B}$ cells. In addition, histological analysis showed that this is mainly due to increased OC number and CTSK activity and decreased OB parameters. More importantly, our work suggests that the RANKL/OPG ratio was increased both in vitro in B-cell culture as well as in vivo in serum and bone specimens. Furthermore, deletion of PKC- $\delta$ selectively in B cells disrupted OC-OB balance by suppressing $\mathrm{OC}$ differentiation and function but co-culture with B-cell supernatant reversed the suppression effect, as well as impairing $\mathrm{OB}$ proliferation and function. To the best of our knowledge, our data showed for the first time a close relationship between PKC- $\delta$ deficient $\mathrm{B}$ cells in the immune system and bone cells in 


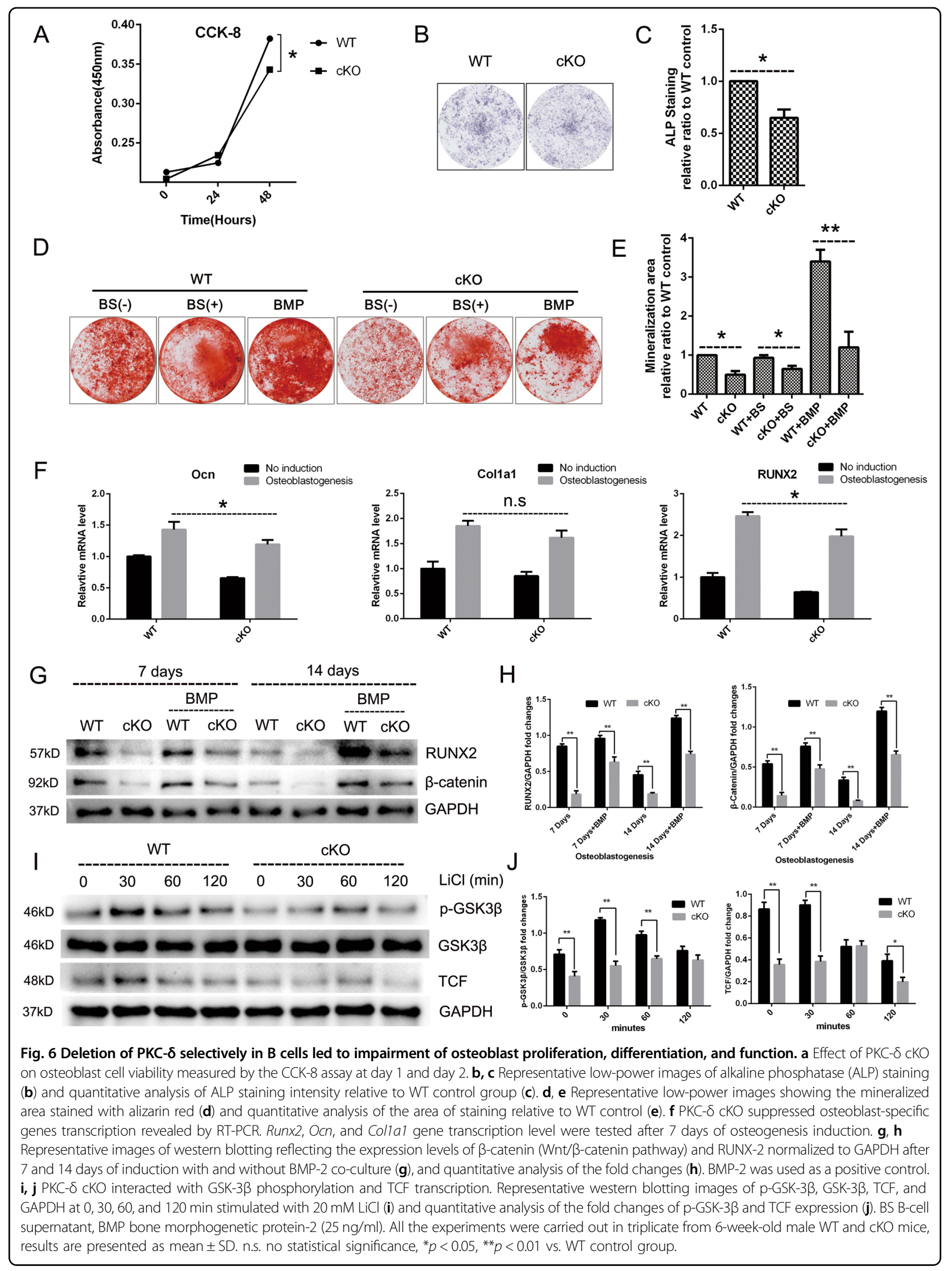


the bone microenvironment, indicating that PKC- $\delta$ may have an important role in the pathogenesis of osteoimmunology-related diseases.

Bone is a dynamic organ formed by deposition and resorption of the bone matrix, which are carried out by $\mathrm{OBs}$ and OCs respectively during bone homeostasis ${ }^{22}$. The most common disorder with this balancing act occurs when the rate of bone resorption exceeds accumulation, resulting in a loss of bone mass, such as is seen in osteoporosis, rheumatoid arthritis and cancer metastasis to bone ${ }^{28}$. It is interesting that changes in the number of B cells are related to osteoporosis. $\mathrm{CD} 19^{+} \mathrm{B}$ cells were low in osteoporotic women and positively correlated to bone mineral density ${ }^{29}$. However, $\mathrm{B}^{2} 20^{+} \mathrm{B}$ lymphocytes were selectively increased 2-4 weeks after ovariectomy and returned to normal when treated with estrogen $^{30}$. Moreover, both estrogen deficiency ${ }^{31}$ and androgen shortage ${ }^{32}$ similarly stimulate B lymphopoiesis, which is involved in the mechanism of stimulated bone resorption. B cells are extensively linked to bone homeostasis from their development in the bone marrow to the homing of terminally differentiated plasma cells back to the bone marrow ${ }^{2}$. The bidirectional regulation of the skeletal system by B cells revolves the RANKL/OPG/ RANK pathway ${ }^{20}$. Our work revealed a close relationship between B-cell RANKL expression and bone remodeling after PKC- $\delta$ ablation in B cells. RANKL is best known for its abundant expression in OBs and osteocytes ${ }^{33,34}$, it is also expressed in bone marrow and lymphoid tissues ${ }^{35}$. However, RANKL expression in immunocyte, including B cells and $\mathrm{T}$ cells, is much lower than that in OBs and osteocytes $^{36}$. Resting $\mathrm{B}$ cells have not been shown to produce significant amounts of RANKL, but activated B cells serve as an important source of RANKL under inflammatory circumstances, such as rheumatoid arthri$\mathrm{tis}^{5}$, periodontitis $\mathrm{s}^{37}$, and HIV induced bone $\operatorname{loss}^{38}$. Our study showed that both B cells and RANKL expression were significantly increased in PKC- $\delta \mathrm{cKO}$ mice, indicating that loss of PKC- $\delta$ had an activation effect on B-cell proliferation. It is interesting that B-cell-specific RANKdeficient mice did not exhibit any discernible osteopetrotic phenotype under physiological conditions ${ }^{39}$. However, RANKL expression by B lymphocytes contributed to ovariectomy-induced bone loss ${ }^{6}$, suggesting that B-cellsderived RANKL does contribute to the increase in OCs and cancellous bone loss that occurs in the pathological state of estrogen loss. All of our results supported the decreased bone mass observed in PKC- $\delta$ cKO mice except for the OC in vitro data, the discrepancy was also existed in PKC- $\delta$ null mice, which exhibited enhanced osteoclastogenesis but increased trabecular bone ${ }^{16}$. The exact reason why the osteoporotic phenotype was accompanied paradoxically with decreased OC differentiation and function in vitro is not clear. We assumed that these conflicting results may be due to an attempt to compensate for the increased RANKL expression in the bone microenvironment as a result of PKC- $\delta$ cKO in B cells. We demonstrated that RANKL was significantly increased both in the serum and in the bone sections in vivo after PKC- $\delta$ ablation. However, when the BMMs were isolated for osteoclastogenesis in vitro and an equal amount of RANKL was applied, it is possible that BMMs from PKC$\delta$ cKO mice, which were pre-exposed to high concentration of RANKL in vivo, became less responsive when stimulated with RANKL in vitro.

Importantly, the positive role that RANKL has in activating the immune system appears to significantly contribute to pathologic bone loss ${ }^{40}$. Furthermore, RANKL has also been demonstrated to play essential roles in lymphocyte development and lymph node organogenesis $^{41,42}$. These observations have spurred intense study of the various ways in which the immune system can influence bone ${ }^{43}$. RANKL exists in two forms: a membranebound form and a soluble form, and both forms were shown to be biologically capable of enhancing OC differentiation ${ }^{44}$. RANKL-RANK signaling also regulates OB differentiation ${ }^{27,45,46}$. Surface-bound RANKL expressed on $\mathrm{OB}$ transferred reverse signals from the exterior of the cell to the interior, which regulated $\mathrm{OBs}^{47}$. Moreover, osteoblastic RANKL functions as a coupling signal acceptor that recognizes vesicular RANK ${ }^{48}$. However, we found that soluble RANKL, unlike membrane-bound RANKL, had no effect on osteoblast formation and function. In our study, we demonstrated that both forms of RANKL were remarkably elevated in mice with PKC- $\delta$ deficiency in B cells by ELISA assay and IHC staining. Moreover, we demonstrated that PKC- $\delta$ deficiency in B cells was accompanied by changes in B-cell number, function, and distribution, especially in the spleen. These results of our study are consistent with previous findings which reported that PKC- $\delta$ negatively regulated B-cell proliferation and played a pivotal role in establishing and controlling antigen-induced B-cell tolerance ${ }^{9,10}$, further attesting that $\mathrm{PKC}-\delta$ signaling plays an important role in B-cell development and RANKL production in B cells.

It is interesting that changes in the bone phenotype and $B$-cell numbers were more pronounced in male mice than in female mice after PKC- $\delta$ ablation in this study. Gender differences are poorly understood and are not well described in the hormone-related-gene deficient mice, such as aromatase-deficient mice ${ }^{49}$ and estrogen receptor knockout mice ${ }^{50}$. However, given that androgen receptor (AR) function is indispensable for male bone formation and remodeling ${ }^{51,52}$, and that androgens regulate PKC- $\delta$ transcription and modulate its apoptotic function in prostate cancer cells ${ }^{53}$, and prenatal testosterone exposure induces hypertension in adult females via an ARdependent PKC- $\delta$-mediated mechanism ${ }^{54}$, it is perhaps 


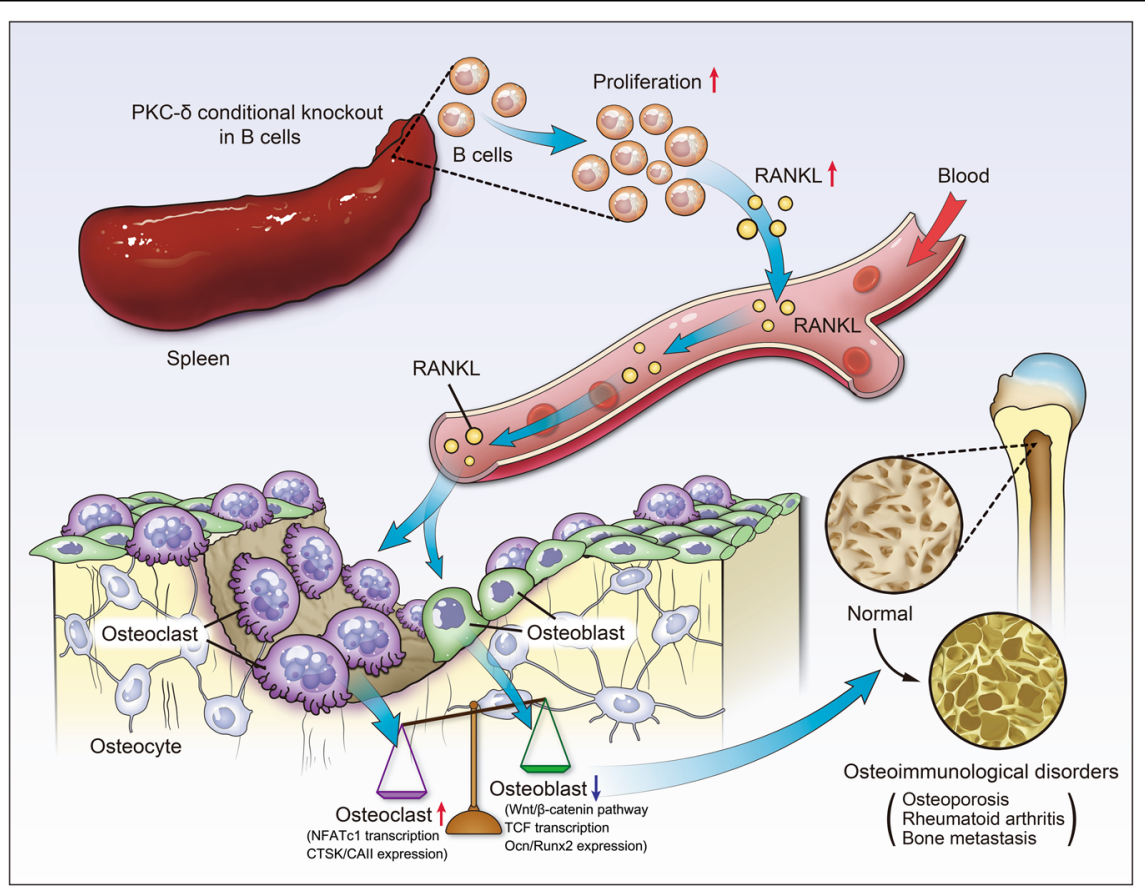

Fig. 7 The schematic model of the hypothesized mechanism by which PKC- $\delta$ ablation selectively in B cells affects bone remodeling. PKC- $\delta$ deficiency in B cells favors bone mass loss, which is owing to the overexpression and secretion of RANKL in these B cells of hyperproliferation and the subsequent osteoclast-osteoblast uncoupling.

not surprising that gender differences were observed in this study. These studies suggest that PKC- $\delta$ has interactions with the AR and lead us to speculate that PKC- $\delta$ may regulate bone mass in a sex dependent manner through AR. However, the precise underlying molecular mechanisms regarding sexual dimorphism need to be further investigated.

In summary, our data clearly demonstrate that mice with PKC- $\delta$ deficiency in B cells favored bone mass loss, which is accompanied by the overexpression and secretion of RANKL in these B cells of hyperproliferation, as well as $\mathrm{OC}-\mathrm{OB}$ uncoupling (schematic model of the hypothesized mechanism was illustrated in Fig. 7). These findings are of great significance for providing novel insights into the tight coupling between PKC- $\delta$ deficient $\mathrm{B}$ cells and bone cells. Detailed molecular understanding of the role of PKC- $\delta$ in both the immune and skeletal systems, as well as providing a molecular basis for developing innovative strategies and therapeutic agents against osteoimmunological disorders, will surely benefit both clinical and basic research in broader disciplines.

\section{Materials and methods}

Animal procedures and animal care

All transgenic mice had a C57BL/6 J and C57BL/6 N genetic background. Mice were group-housed under conventional conditions: 12 -h light/dark cycle, standard rodent chow and water ad libitum in standard cages. All mice were maintained at National Resource Center for Mutant Mice Model Animal Research Center of Nanjing University in China according to institutional guidelines. All the experiments were approved by the Institutional Animal Care and Use Committee of The Third Affiliated Hospital of Sun Yat-sen University (Approval No: IACUC-F3-18-1202) and were performed according to EU Directive 2010/63/EU.

\section{Generation of B-cell-specific PKC- $\delta$ conditional knockout mice}

LoxP mice were purchased from RIKEN BioResource Research Center (Stock Number: RBRC06462, Strain Name: C57BL/6-Prkcd $<$ tm1Shb $>$, 3-1-1 Koyadai, Tsukuba, Ibaraki 305-0074, Japan), and CD19-Cre mice were purchased from The Jackson Laboratory (Stock Number: 006785, Strain Name: B6.129P2(C)-CD19 $9^{\mathrm{tm} 1(\mathrm{cre}) \mathrm{Cgn}} / \mathrm{J}$, Bar Harbor, ME, USA). Mice with B-cell-selective knockout of the PKC- $\delta\left(\mathrm{CD} 19-\mathrm{Cre}^{+} \mathrm{PKC}-\delta^{\text {flox/flox }}\right)$ were generated by crossing mice heterozygous for a floxed exon 7 PKC- $\delta$ allele (PKC- $\delta^{(\mathrm{ex} 7) \text { flox/+ }}$ ) with heterozygous mice carrying a cyclization recombinase of which the expression is controlled by the CD19 promoter (CD19-Cre $\left.{ }^{+} \mathrm{PKC}-\delta^{\text {flox/++}}\right)$. Offspring mice were weaned at 3 weeks of age and genotyped by PCR, the presence of the CD19-Cre transgene was determined on genomic DNA via PCR with forward primer: 5'-ATTTGCCTGCATTACCGGTC-3' and reverse primer: $5^{\prime}$-ATCAACGTTTTCTTTTCGG-3' 
resulting in a 377 bp fragment. CD19-Cre ${ }^{-} \mathrm{PKC}-\delta^{\text {flox/flox }}$ littermate mice were used as control.

\section{Micro-CT measurement}

Microstructure of bone in mice were measured by highresolution micro-CT as previously described ${ }^{55,56}$. MicroCT scanning was performed in vitro on the right tibia and femur using a Scanco $\mu$ CT100 scanner (Brüttisellen, Zurich, Switzerland) with a fixed isotropic voxel size of $10 \mu \mathrm{m}$, 100 slices, $70 \mathrm{kV}$ at $200 \mu \mathrm{A}, 300 \mathrm{~ms}$ integration time. Micro-CT parameters were evaluated in the trabecular region of the distal femur and proximal tibia, commencing at a distance of $0.5 \mathrm{~mm}$ from the growth plate and extending a further $1.5 \mathrm{~mm}$ proximally for femur and distally for tibia. Parameters assessed included bone volume fraction (bone volume/total volume, BV/TV, \%), trabecular number (Tb.N, $\mathrm{mm}^{-1}$ ), trabecular thickness (Tb.Th, mm) and trabecular separation (Tb.Sp, mm). Cortical bone was analyzed starting at a distance of $2.75 \mathrm{~mm}$ from the growth plate and extending $1 \mathrm{~mm}$ towards the mid shaft to determine total cortical area (Tt. $\left.\mathrm{Ar}, \mathrm{mm}^{2}\right)$, cortical bone area $\left(\mathrm{Ct} . \mathrm{Ar}, \mathrm{mm}^{2}\right)$, cortical area fraction (Ct.Ar/Tt.Ar, \%), and cortical thickness (Ct.Th, $\mu \mathrm{m}$ ).

\section{Bone histomorphometry and immunohistochemistry}

Left tibias were fixed overnight in $10 \%$ buffered formalin, decalcified with 14\% EDTA for 7 days, embedded in paraffin and histological sections of $3 \mu \mathrm{m}$ were then prepared for staining. Trabecular bone parameters and in vivo OB parameters were assessed from $H \& E$ stained sections as described ${ }^{57}$, while in vivo OC parameters were obtained from TRAP staining as previously ${ }^{58}$. Trabecular bone region of interest was measured $500 \mu \mathrm{m}$ below the growth plate and $1 \mathrm{~mm}$ in height at the proximal tibia. Histomorphometric analysis was performed by quantifying parameters including osteoclast surface per bone surface (Oc.S/BS), number of osteoclasts per bone perimeter (N.Oc/B.Pm), osteoblast surface per bone surface (Ob.S/BS) and number of osteoblasts per bone perimeter (N.Ob/B.Pm) using an Olympus microscope and the BIOQUANT OSTEO software (BIOQUANT OSTEO 2013 Ver.13.20.6, Nashville, TN, USA). We counted the numbers of positively stained cells in five sequential sections per mouse in each group and normalized them to the number per millimeter of adjacent bone surface or per square millimeter.

For bone tissue IHC, antigen retrieval was carried out by incubating specimens with bone tissue specific antigenretrieval solutions (SBT100013, Showbio, Shanghai, China) for $60 \mathrm{~min}$ at $37^{\circ} \mathrm{C}$ before commencing with IHC staining protocol. Non-specific binding was blocked with goat serum for $30 \mathrm{~min}$ at room temperature before incubation with primary antibody (CTSK ab19027, RANKL ab45039, OPG ab9986 and osteocalcin ab93876. Abcam, Cambridge, UK) against mouse antigens at $4{ }^{\circ} \mathrm{C}$ overnight in a humidified container. For detection, sections were treated with HRP-conjugated secondary antibody (GK500505A, Dako, Carpinteria, CA, USA) for $30 \mathrm{~min}$ at $37^{\circ} \mathrm{C}$, followed by DAB substrate (ZLI-9017, ZSGB-Bio, Beijing, China) for 30 seconds, counterstained with Mayer's hematoxylin, dehydrated, and mounted. For negative controls, sections were treated with the same amount of primary antibody diluent without primary antibodies. Semi-quantitative evaluation of CTSK, RANKL and OPG was performed as previously described ${ }^{59}$ in five random trabecular regions (mainly OCs and OBs) of each section. Briefly, staining intensity (A) was classified as 0 (negative), 1 (weak), 2 (moderate) and 3 (strong). The percentage of positive cells (B) examined in 100 cells were divided into 0 (<5\%), 1 (5-25\%), 2 (26-50\%), 3 (51-75\%) and $4(>75 \%)$. The IHC result (possibly $0,1,2,3,4,6,8,9,12$ ) was the product of $\mathrm{A}$ and $\mathrm{B}$ and classified as 0 (equal to negative $(-)$ ); 1-4 (weakly positive $(+)$ ); 5-8 (moderately positive $(++))$ and 9-12 (strongly positive $(+++))$. Two experienced pathologists scored each section, they were blinded to the identity of each specimen at the time they determined the score and the final scores were evaluated by consensus.

Safranin O Fast Green Staining, Masson's trichrome staining and Von Kossa staining (in undecalcified sections) were used to assess chondrocytes, organic, and inorganic matrix components, respectively. Von Kossa staining was performed by incubating sagittal nondecalcified sections $(4 \mu \mathrm{m})$ of the left femur with $1 \%$ silver nitrate solution under ultraviolet light.

\section{Real-time RT-PCR assay}

Total RNA was isolated with a total RNA extraction kit (R6834, Omega, USA) and reverse transcription was carried out using $1 \mu \mathrm{g}$ of total RNA with reverse transcriptase (RR036, Takara Bio, Japan) in a volume of $20 \mu \mathrm{l}$ according to the manufacturer's instructions. One microliter of cDNA was amplified with the specific primers (Invitrogen) as follows and were quantified on a Light Cycler 480II (Roche) using multiple kits (4887352001, SYBR Green I Master, Roche), normalizing with GAPDH. PCR primers were: mouse $p k c-\delta, 5^{\prime}-\mathrm{A}$ CCCAGAAGACTGTGGATGG-3' (forward) and 5- CGT CCCTGTCTAGCATCACA-3' (reverse); mouse nfatc1, 5'-CCGTTGCTTCCAGAAAATAACA-3' (forward) and $5^{\prime}$ - TGTGGGATGTGAACTCGGAA-3' (reverse); mouse ctsk, 5'-CCAGTGGGAGCTATGGAAGA-3' (forward) and $5^{\prime}$ - AAGTGGTTCATGGCCAGTTC-3' (reverse); mouse calcitonin receptor, 5'-TGCAGACAACTCTTGG TTGG-3' (forward) and 5'- TCGGTTTCTTCTCCTC TGGA-3' (reverse); mouse ocn, 5'-GCGCTCTGTCT CTCTGACCT- $3^{\prime}$ (forward) and $5^{\prime}$-ACCTTATTGCC 
CTCCTGCTT-3' (reverse); mouse colla1, 5'-CT GGCGGTTCAGGTCCAAT-3' (forward) and 5'- TCC AAACCACTGAAGCCTCG-3' (reverse); mouse runx2, 5'-CGCATTCCTCATCCCAGTAT-3' (forward) and 5'TGTAGGTAAAGGTGGCTGGG-3' (reverse); mouse gapdh, $5^{\prime}$-CACATTGGGGGTAGGAACAC-3' (forward) and 5'-TGAGTCCTTCCACGATACCAAAGTT-3' (reverse); all experiments were repeated three times. The $\mathrm{Ct}$ value of the reference gene GAPDH was subtracted from the Ct value of the target genes $(\Delta \mathrm{Ct})$, and the average $\Delta \mathrm{Ct}$ value of triplicates was taken. Relative expression levels of each gene were obtained by using the $2^{-\Delta \Delta \mathrm{Ct}}$ method.

\section{Western blotting assay}

Cells were lysed in RIPA buffer containing $20 \mathrm{nM}$ Tris$\mathrm{HCl}, 150 \mathrm{mM} \mathrm{NaCl}, 1 \%$ Triton X-100, 0.2\% deoxycholate, protease, and phosphatase inhibitors for $30 \mathrm{~min}$ on ice for protein extraction. Protein concentrations of cell lysates were determined by using a BCA assay. An equal amount of proteins $(30 \mu \mathrm{g} /$ lane $)$ was resolved by SDSpolyacrylamide gel electrophoresis and then transferred to the PVDF membrane (Millipore, Boston, MA, USA). The membrane was probed with the indicated primary antibodies overnight at $4{ }^{\circ} \mathrm{C}$. Antibodies were purchased from Abcam (PKC- $\delta$, ab182126; CAII, ab124687), Cell Signaling Technologies (GSK-3 $\beta$, \#12456; phospho-GSK3ß, \#5558; TCF, \#2203; RUNX-2, \#12556; $\beta$-catenin, \#8480) and SANTA CRUZ (CTSK, sc-48353; NFATc1, sc-7294; GAPDH, sc-66163; $\alpha$-Tubulin, sc-69970). Blots were then developed using HRP-conjugated secondary antibodies for $60 \mathrm{~min}$ at room temperature and were visualized by using enhanced ECL reagents (Amersham, Pittsburgh, PA, USA) according to manufacturer's instructions. Images were acquired on an Imagequant LAS 4000. $\alpha$-Tubulin or GAPDH was used as a loading control to ensure equal amounts of protein were loaded per lane. Experiments were repeated three times and quantification of all the blots is presented as mean \pm SD. Signal intensities were quantified by NIH ImageJ software.

\section{B-cell isolation, purification, and culture}

For primary B-cell purification, single-cell suspensions were prepared from pooled spleens of WT and PKC- $\delta$ cKO mice. Cell suspensions were depleted of red blood cells by ammonium chloride lysis buffer (containing $0.15 \mathrm{M} \mathrm{NH} 4 \mathrm{Cl}, 10 \mathrm{mM}$ Tris-HCl, $0.1 \mathrm{mM}$ EDTA). B cells were purified using the B-Cell Isolation Kit (MiltenyiBiotec, 130-121-301) according to the protocol of the manufacturer. The purity of B cells was more than $95 \%$, which was confirmed by flow-cytometric analysis using anti-CD19 antibody (BD Biosciences, Catalog \# 550992).

For in vitro B-cell culture, enriched primary mouse B cells were cultured in RPMI 1640 (HyClone) containing $10 \% \mathrm{FBS}, 50 \mathrm{nM}$ 2-ME, $4 \mathrm{mM}$ l-glutamine, and $1 \%$ penicillin/streptomycin. Cells were stimulated with $4 \mu \mathrm{g} / \mathrm{ml}$ CpGODN 2006 (CPG, Invivogen) and $1 \mu \mathrm{g} / \mathrm{ml}$ trimeric CD40L (R\&D systems). Phor-bol-12-myristate-13acetate (PMA, $50 \mathrm{ng} / \mathrm{ml}$, Sigma), ionomycin $(500 \mathrm{ng} / \mathrm{ml}$, Sigma) and Brefeldin A (BFA, $10 \mathrm{mg} / \mathrm{ml}$, Sigma) were added at the indicated concentrations at the last $6 \mathrm{~h}$.

For preparation of B-cell supernatant for $\mathrm{OB}$ and $\mathrm{OC}$ co-cultures, mouse $\mathrm{B}$ cells were purified from splenocytes using the B-cell isolation kit and $2 \times 10^{6}$ cells $/ \mathrm{ml}$ grown in RPMI 1640 supplemented with 10\% FBS, CPG and CD40L. This growth medium was collected at day 4 as B-cell supernatants. The supernatants were concentrated 10-fold through Amicon Ultra-15 centrifugal filter devices with a NMWL-3KD device (Millipore, Billerica, MA, USA) before use. For the co-culture experiments, $25 \mu \mathrm{l} \mathrm{B-}$ cell supernatant per milliliter complete culture medium was used as described ${ }^{60}$.

\section{Flow-cytometry assay}

For phenotypic analysis, single-cell suspensions were prepared from spleen, lymph nodes, liver and thymus. Briefly, lymph nodes and thymus were passed through a 200-gauge steel mesh and washed with PBS. Spleens were firstly passed through a 200-gauge steel mesh and then RBC were lysed and remaining cells washed with PBS. Livers were passed through a 200-gauge steel mesh and the cell pellets were collected in the centrifuge tube, the mononuclear cells in the pellets were isolated by gradient centrifugation with $40 \%$ and $70 \%$ Percoll. Single-cell suspensions were stained according to the manufacturers' instructions using fluorochrome-coupled monoclonal antibodies (mAbs). The following $\mathrm{mAbs}$ were obtained from BD Bioscience: BV421-conjugated anti-CD45.2 (Catalog \# 562895), PE-conjugated anti-CD5 (Catalog \# 553022), APC-conjugated anti-CD19 (Catalog \# 550992). PE-Cy7-conjugated anti-IL-10 (Catalog \# 505026) was purchased from Biolegend. Flow-cytometric analyses were performed using an LSR II flow cytometer (BD Biosciences) and flow-cytometry data were analyzed using Flowjo V10 software (BD Biosciences).

\section{Cryosectioning and immunofluorescent assay}

Spleens were harvested, fixed with $4 \%$ paraformaldehyde, washed in PBS, and dehydrated overnight in 30\% sucrose (Sigma). The samples were embedded in TissueTekOCT compound (Bio-Optica) and frozen in an ethanol dry ice bath. Sections of $7 \mu \mathrm{m}$ were placed on glass slides (Bio-Optica) and blocked for $30 \mathrm{~min}$ with PBSTween $0.05 \%$ plus $0.5 \%$ FBS. Sections were stained with Abs directed against B220 (Alexa Fluor 594, Biolegend, 103254, San Diego, CA, USA) for $1 \mathrm{~h}$ at $37^{\circ} \mathrm{C}$ to visualize B cells, nuclei were counterstained using DAPI (KGA215, Jetway, Nanjing, China) for another $15 \mathrm{~min}$ at $37^{\circ} \mathrm{C}$, then coverslips were mounted on a microscope slide with 
Antifade Mounting Medium (P0126, Beyotime, Jiangsu, China). Finally, Images were acquired using a laser scanning confocal microscopy (LSM880, Zeiss, Jena, Germany).

\section{ELISA assay}

Mouse blood was obtained from the Fundus vein. Samples were allowed to clot for $2 \mathrm{~h}$ at room temperature and then centrifuged for $15 \mathrm{~min}$ at $12,000 \mathrm{rpm}$ at $4{ }^{\circ} \mathrm{C}$. Serum was collected and frozen at $-20^{\circ} \mathrm{C}$ until use. OPG (Catalog \# EMTNFRSF11B) and RANKL (Catalog \# EMTNFSF11) ELISA kits were purchased from Invitrogen, while $\beta$-CTX (Catalog \# E-EL-M0372c) and PINP (Catalog \# E-ELM0233c) ELISA kits were obtained from Elabscience. Serum OPG, RANKL, $\beta$-CTX and PINP produced in vivo by WT and PKC- $\delta$ cKO mice were measured by ELISA assay according to the manufacturer's instructions. All the optical densities (ODs) measured after reactions were converted to the concentration using their standard curves. All the samples were measured in triplicate.

\section{Macrophage isolation from mouse bone marrow, osteoclastogenesis assay, and tartrate-resistant acid phosphatase staining}

Bone marrow from tibia and femur of WT and cKO mice was flushed with $\alpha$-MEM and cells pelleted by centrifugation at $1500 \mathrm{rpm}$ for $5 \mathrm{~min}$. Bone marrow cells were then cultured in complete $\alpha$-MEM media supplemented with $10 \%$ fetal bovine serum, $1 \%$ antibiotic, and $30 \mathrm{ng} / \mathrm{ml}$ of recombinant mouse macrophage colony stimulating factor (MCSF, 416-ML, R\&D systems, Minneapolis, MN, USA) at $37{ }^{\circ} \mathrm{C}$ in a humidified $5 \% \mathrm{CO}_{2}$ atmosphere to allow cell attachment. After 2 days, nonadherent cells were removed and discarded; adherent cells were cultured to confluence and treated as bone marrow macrophages (BMMs). BMMs of passage 1 or 2 were used in the experiments below. BMMs were seeded on to 96well plate $\left(6 \times 10^{3}\right.$ cells/well $)$ and treated with $30 \mathrm{ng} / \mathrm{ml}$ MCSF, and 100/25 ng/ml RANKL (462-TEC, R\&D systems) to induce OC formation. The media was replaced with supplemented MCSF and RANKL (with and without BS) every 2 days and after 7 days of culture, the cells were fixed in $2.5 \%$ glutaraldehyde and stained with Acid Phosphatase staining kit (387 A, Sigma-Aldrich, St. Louis, MO, USA) according to the protocol of the manufacturer. TRAP-positive multinucleated cells with more than three nuclei were counted as OCs using light microscopy. The number of OCs in each well was counted using ImageJ software.

\section{Hydroxyapatite resorption assay}

The diluted BMMs cell suspension $\left(100 \mu \mathrm{l}, 5 \times 10^{3}\right.$ cells per well) was transferred into each well of a 96-well Corning Osteo Assay Surface plate (3989, Corning Life
Sciences, Tewksbury, MA, USA) coated by hydroxyapatite to begin the differentiation process. Plates were incubated at $37{ }^{\circ} \mathrm{C}$ in a humidified atmosphere of $5 \% \mathrm{CO}_{2}$ with differentiation medium $(30 \mathrm{ng} / \mathrm{ml}$ MCSF combined $100 \mathrm{ng} / \mathrm{ml}$ RANKL) (with and without BS) changed every 3 days. After 7 days, the plates were stripped with 1.2\% sodium hypochlorite solution for $5 \mathrm{~min}$ to remove cells, rinsed with distilled water, and air-dried prior to imaging. Overlapping images of the entire well surface were taken at $\times 20$ magnification and these were then used to produce a composite image using Image Composite Editor (ICE 2.0, Microsoft, USA). The total resorption area was then measured in the composite image using Image-Pro Plus (version 6.0, Media Cybernetics Company, Rockville, MD, USA).

\section{In vitro osteoblast differentiation assay and CCK-8 measurement of osteoblast proliferation}

OB precursors were obtained from calvaria of 6-week-old mice after digesting with collagenase II solutions as described previously ${ }^{61}$. Cells were seeded at a density of $1 \times$ $10^{6}$ cells $/ \mathrm{ml}$ in complete Dulbecco's Modified Eagle's Medium (DMEM) supplemented with 10\% FBS, 2mM L-glutamine, $1 \%$ penicillin and streptomycin. Osteogenic media $\left(10^{-8} \mathrm{M}\right.$ dexamethasone, $10 \mathrm{mM} \beta$-glycerophosphate and $50 \mu \mathrm{g} / \mathrm{ml}$ ascorbic acid) was used for culturing osteoblasts. To detect osteoblastogenesis short-time course signaling, $20 \mathrm{mM} \mathrm{LiCl}$ (Sigma, Lot \# MKBZ4804V) was used as previously described ${ }^{62}$. BS co-culture and BMP-2 (novoprotein, Catalog \#C012, Shanghai, China) stimulation $(25 \mathrm{ng} / \mathrm{ml})$ were added at the indicated time-points.

Passage two OBs were cultured at $2.5 \times 10^{3}$ cells/well in a 96-well plate for $24 \mathrm{~h}$. To evaluate the effect of PKC- $\delta$ $\mathrm{cKO}$ on $\mathrm{OB}$ viability, proliferation was measured at 24 and $48 \mathrm{~h}$ with the Cell Counting Kit-8 (CCK-8) kit (Dojindo Molecular Technologies, Inc., Kumamoto, Japan) after culture according to the manufacturer's protocol. At the end of culture, $10 \mu \mathrm{l}$ of CKK-8 was added to each well, cells were cultured for an additional $1 \mathrm{~h}$, absorbance was measured at $450 \mathrm{~nm}$ using a micro plate reader (BioTek ELx800, BioTek Inc., Winooski, VT, USA). The experiments were performed in triplicate.

\section{Alkaline phosphatase staining, Alizarin red staining, and mineralization}

ALP was considered as an early differentiation marker of OBs while mineralization activity occurred in the later stage. ALP staining kit (BCIP/NBT Liquid Substrate System, Catalog \# B1911, Sigma) was used to test the activity of ALP on days 7 in the 48-well plates according to the manufacturer's protocols. Alizarin red staining kit (A5533, Sigma) was used to test the mineralization function of OBs on day 21 in 24-well plates. The ALP staining intensity and the mineralized area were visualized 
using ELISPOT analyzer (S6 ULTRA, C. T. L, USA) and measured by ImageJ software.

\section{Statistical analyses}

All data presented in this study are representative of one of three independent experiments, and the results are presented as mean \pm SD. Statistical analyses were performed with the two-tailed Student's $t$-test for two-group comparisons, or one-way ANOVA and post-hoc multiple comparisons with Bonfer-roni correction for three- or four-group comparisons. All data analysis was performed with SPSS 20.0 Package (SPSS software 20.0; SPSS, Chicago, IL, USA). All statistical tests were two-sided and values of $p$ smaller than 0.05 were considered significant.

\section{Acknowledgements}

This study was supported by grant from the Guangdong Natural Science Foundation (No. 2018A030313248), the National Natural Science Foundation of China (No. 81772398), and the Fundamental Research Funds for the Central Universities (No. 17ykpy49). This study was supported in part by the Australian National Health and Medical Research Council (NHMRC, No. 1107828), University of Western Australia Research Collaboration Awards. We sincerely thank to Professor Xiaojuan Li from Southern Medical University for her helpful comments on this study. Thanks to Dr. Samuel Bennett, The University of Western Australia, for assistance with editing of the manuscript.

\section{Author details}

'Department of Spine Surgery, The Third Affiliated Hospital of Sun Yat-sen University, Guangzhou Guangdong, China. ${ }^{2}$ Guangdong Provincial Center for Quality Control of Minimally Invasive Spine Surgery, Guangzhou, China. ${ }^{3}$ Guangdong Provincial Center for Engineering and Technology Research of Minimally Invasive Spine Surgery, Guangzhou, China. ${ }^{4}$ The Biotherapy Center, The Third Affiliated Hospital of Sun Yat-sen University, Guangzhou Guangdong, China. ${ }^{5}$ School of Biomedical Sciences, The University of Western Australia, Perth, WA, Australia. 'Department of Internal Medicine, The Ohio State University Wexner Medical Center, Columbus, OH, USA. 'Laboratory of Antiinflammatory and Immunomodulatory Pharmacology, Southern Medical University, Guangzhou Guangdong, China

\section{Conflict of interest}

The authors declare that they have no conflict of interest.

\section{Publisher's note}

Springer Nature remains neutral with regard to jurisdictional claims in published maps and institutional affiliations.

Supplementary Information accompanies this paper at (https://doi.org/ 10.1038/s41419-020-02947-3).

Received: 16 December 2019 Revised: 25 June 2020 Accepted: 29 June 2020

Published online: 16 September 2020

\section{References}

1. Arron, J. R. \& Choi, Y. Bone versus immune system. Nature $\mathbf{4 0 8}, 535-536$ (2000).

2. Horowitz, M. C., Fretz, J. A. \& Lorenzo, J. A. How B cells influence bone biology in health and disease. Bone 47, 472-479 (2010).

3. Meednu, N. et al. Production of RANKL by memory B cells: a link between $B$ cells and bone erosion in rheumatoid arthritis. Arthritis Rheumatol. 68, 805-816 (2016).
4. Li, Y. et al. B cells and T cells are critical for the preservation of bone homeostasis and attainment of peak bone mass in vivo. Blood 109, 3839-3848 (2007).

5. Yeo, L. et al. Cytokine mRNA profiling identifies $B$ cells as a major source of RANKL in rheumatoid arthritis. Ann. Rheum. Dis. 70, 2022-2028 (2011).

6. Onal, M. et al. Receptor activator of nuclear factor kappaB ligand (RANKL) protein expression by B lymphocytes contributes to ovariectomy-induced bone loss. J. Biol. Chem. 287, 29851-29860 (2012).

7. Ono, Y. et al. The structure, expression, and properties of additional members of the protein kinase C family. J. Biol. Chem. 263, 6927-6932 (1988).

8. Salzer, E., Santos-Valente, E., Keller, B., Warnatz, K. \& Boztug, K. Protein kinase C delta: a gatekeeper of immune homeostasis. J. Clin. Immunol. 36, 631-640 (2016).

9. Mecklenbrauker, I., Saijo, K., Zheng, N. Y., Leitges, M. \& Tarakhovsky, A. Protein kinase Cdelta controls self-antigen-induced B-cell tolerance. Nature 416, 860-865 (2002).

10. Miyamoto, A. et al. Increased proliferation of B cells and auto-immunity in mice lacking protein kinase Cdelta. Nature 416, 865-869 (2002).

11. Kuehn, H. S. et al. Loss-offfunction of the protein kinase C delta (PKCdelta) causes a B-cell lymphoproliferative syndrome in humans. Blood 121 3117-3125 (2013).

12. Salzer, E. et al. B-cell deficiency and severe autoimmunity caused by deficiency of protein kinase C delta. Blood 121, 3112-3116 (2013).

13. Belot, A. et al. Protein kinase cdelta deficiency causes mendelian systemic lupus erythematosus with B cell-defective apoptosis and hyperproliferation. Arthritis Rheumatism 65, 2161-2171 (2013).

14. Nanthapisal, S. et al. Early-onset juvenile SLE associated with a novel mutation in protein kinase C delta. Pediatrics. 139, e20160781 (2017).

15. Cremasco, V. et al. Protein kinase C-delta deficiency perturbs bone homeostasis by selective uncoupling of cathepsin $\mathrm{K}$ secretion and ruffled border formation in osteoclasts. J. Bone Miner. Res. 27, 2452-2463 (2012).

16. Khor, E. C. et al. Loss of protein kinase C-delta protects against LPS-induced osteolysis owing to an intrinsic defect in osteoclastic bone resorption. PLoS ONE 8, e70815 (2013).

17. Ueno, M. et al. Protein kinase $\mathrm{C}$ modulates the synthesis of nitric oxide by osteoblasts. Calcif. Tissue Int. 63, 22-26 (1998).

18. Tu, X. et al. Noncanonical Wnt signaling through G protein-linked PKCdelta activation promotes bone formation. Dev. cell 12, 113-127 (2007).

19. Yang, $X$. et al. Protein kinase $C$ delta null mice exhibit structural alterations in articular surface, intra-articular and subchondral compartments. Arthritis Res. Ther. 17, 210 (2015).

20. Okamoto, K. et al. Osteoimmunology: the conceptual framework unifying the immune and skeletal systems. Physiol. Rev. 97, 1295-1349 (2017).

21. Manabe, N. et al. Connection between B lymphocyte and osteoclast differentiation pathways. J. Immunol. 167, 2625-2631 (2001).

22. Raggatt, L. J. \& Partridge, N. C. Cellular and molecular mechanisms of bone remodeling. J. Biol. Chem. 285, 25103-25108 (2010).

23. Matta, C. \& Mobasheri, A. Regulation of chondrogenesis by protein kinase C: emerging new roles in calcium signalling. Cell. Signal. 26, 979-1000 (2014).

24. Paiva, K. B. \& Granjeiro, J. M. Bone tissue remodeling and development: focus on matrix metalloproteinase functions. Arch. Biochem. Biophys. 561, 74-87 (2014).

25. Deng, J. et al. B1a cells play a pathogenic role in the development of autoimmune arthritis. Oncotarget 7, 19299-19311 (2016).

26. Boyle, W. J., Simonet, W. S. \& Lacey, D. L. Osteoclast differentiation and activation. Nature 423, 337-342 (2003).

27. Cao, X. RANKL-RANK signaling regulates osteoblast differentiation and bone formation. Bone Res. 6, 35 (2018).

28. Zaidi, M. Skeletal remodeling in health and disease. Nat. Med. 13, 791-801 (2007).

29. Breuil, V. et al. Immune changes in post-menopausal osteoporosis: the immunos study. Osteoporos. Int. 21, 805-814 (2010).

30. Masuzawa, T. et al. Estrogen deficiency stimulates B lymphopoiesis in mouse bone marrow. J. Clin. Invest. 94, 1090-1097 (1994).

31. Miyaura, C. et al. Increased B-lymphopoiesis by interleukin 7 induces bone loss in mice with intact ovarian function: similarity to estrogen deficiency. Proc. Nat Acad. Sci. USA 94, 9360-9365 (1997).

32. Onoe, $Y$. et al. Comparative effects of estrogen and raloxifene on B lymphopoiesis and bone loss induced by sex steroid deficiency in mice. J. Bone Miner. Res. 15, 541-549 (2000). 
33. Schaffler, M. B., Cheung, W. Y., Majeska, R. \& Kennedy, O. Osteocytes: master orchestrators of bone. Calcif. Tissue Int. 94, 5-24 (2014).

34. Tu, X. et al. Osteocytes mediate the anabolic actions of canonical Wnt/ beta-catenin signaling in bone. Proc. Natl Acad. Sci. USA 112, E478-E486 (2015).

35. Takayanagi, H. Osteoimmunology: shared mechanisms and crosstalk between the immune and bone systems. Nat. Rev. Immunol. 7, 292-304 (2007).

36. Nakashima, T. et al. Evidence for osteocyte regulation of bone homeostasis through RANKL expression. Nat. Med. 17, 1231-1234 (2011).

37. Kawai, T. et al. B and T lymphocytes are the primary sources of RANKL in the bone resorptive lesion of periodontal disease. Am. J. Pathol. 169, 987-998 (2006).

38. Titanji, K. Beyond antibodies: B cells and the OPG/RANK-RANKL pathway in health, non-HIV disease and HIV-induced bone loss. Front. Immunol. 8, 1851 (2017).

39. Perlot, T. \& Penninger, J. M. Development and function of murine B cells lacking RANK. J. Immunol. 188, 1201-1205 (2012).

40. Terashima, A. \& Takayanagi, H. Overview of osteoimmunology. Calcif. Tissue Int. 102, 503-511 (2018).

41. Walsh, M. C. et al. Osteoimmunology: interplay between the immune system and bone metabolism. Annu. Rev. Immunol. 24, 33-63 (2006).

42. Kong, Y. Y. et al. OPGL is a key regulator of osteoclastogenesis, lymphocyte development and lymph-node organogenesis. Nature 397, 315-323 (1999).

43. Takayanagi, $\mathrm{H}$. Osteoimmunology and the effects of the immune system on bone. Nat. Rev. Rheumatol. 5, 667-676 (2009).

44. Xiong, J. et al. Soluble RANKL contributes to osteoclast formation in adult mice but not ovariectomy-induced bone loss. Nat. Commun. 9, 2909 (2018).

45. Kong, $\mathrm{N}$. et al. Induced T regulatory cells suppress osteoclastogenesis and bone erosion in collagen-induced arthritis better than natural $T$ regulatory cells. Ann. Rheum. Dis. 71, 1567-1572 (2012).

46. Luo, Y. et al. Human gingival tissue-derived MSC suppress osteoclastogenesis and bone erosion via CD39-adenosine signal pathway in autoimmune arthritis. EBioMedicine 43, 620-631 (2019).

47. Zhang, S. et al. Osteoclast regulation of osteoblasts via RANK-RANKL reverse signal transduction in vitro. Mol. Med. Rep. 16, 3994-4000 (2017).

48. Ikebuchi, Y. et al. Coupling of bone resorption and formation by RANKL reverse signalling. Nature 561, 195-200 (2018).

49. Oz, O. K. et al. Bone has a sexually dimorphic response to aromatase deficiency. J. Bone Miner. Res. 15, 507-514 (2000).
50. Sims, N. A. et al. Deletion of estrogen receptors reveals a regulatory role for estrogen receptors-beta in bone remodeling in females but not in males. Bone 30, 18-25 (2002).

51. Wang, J. \& Stern, P. H. Sex-specific effects of estrogen and androgen on gene expression in human monocyte-derived osteoclasts. J. Cell. Biochem. 112 3714-3721 (2011)

52. Pihlajamaa, P., Sahu, B. \& Janne, O. A. Determinants of receptor- and tissuespecific actions in androgen signaling. Endocr. Rev. 36, 357-384 (2015).

53. Gavrielides, M. V., Gonzalez-Guerrico, A. M., Riobo, N. A. \& Kazanietz, M. G. Androgens regulate protein kinase Cdelta transcription and modulate its apoptotic function in prostate cancer cells. Cancer Res. 66, 11792-11801 (2006).

54. Blesson, C. S., Chinnathambi, V., Hankins, G. D., Yallampalli, C. \& Sathishkumar, K. Prenatal testosterone exposure induces hypertension in adult females via androgen receptor-dependent protein kinase Cdelta-mediated mechanism. Hypertension 65, 683-690 (2015).

55. Jadhav, G., Teguh, D., Kenny, J., Tickner, J. \& Xu, J. Morc3 mutant mice exhibit reduced cortical area and thickness, accompanied by altered haematopoietic stem cells niche and bone cell differentiation. Sci. Rep. 6, 25964 (2016)

56. Kular, J. et al. Choline kinase beta mutant mice exhibit reduced phosphocholine, elevated osteoclast activity, and low bone mass. J. Biol. Chem. 290 , 1729-1742 (2015)

57. Kim, $\mathrm{H}$. et al. IgSF11 regulates osteoclast differentiation through association with the scaffold protein PSD-95. Bone Res. 8, 5 (2020).

58. Sawyer, A., Lott, P. Titrud, J. \& McDonald, J. Quantification of tartrate resistant acid phosphatase distribution in mouse tibiae using image analysis. Biotech. Histochem. 78, 271-278 (2003).

59. Da, C. L., Xin, Y., Zhao, J. \& Luo, X. D. Significance and relationship between Yes-associated protein and survivin expression in gastric carcinoma and precancerous lesions. World J. Gastroenterol. 15, 4055-4061 (2009).

60. Bai, L. et al. Hepatocyte growth factor mediates mesenchymal stem cellinduced recovery in multiple sclerosis models. Nat. Neurosci. 15, 862-870 (2012).

61. Bakker, A. D. \& Klein-Nulend, J. Osteoblast isolation from murine calvaria and long bones. Methods Mol. Biol. 816, 19-29 (2012).

62. Li, J. et al. Dexamethasone shifts bone marrow stromal cells from osteoblasts to adipocytes by C/EBPalpha promoter methylation. Cell Death Dis. 4, e832 (2013). 